
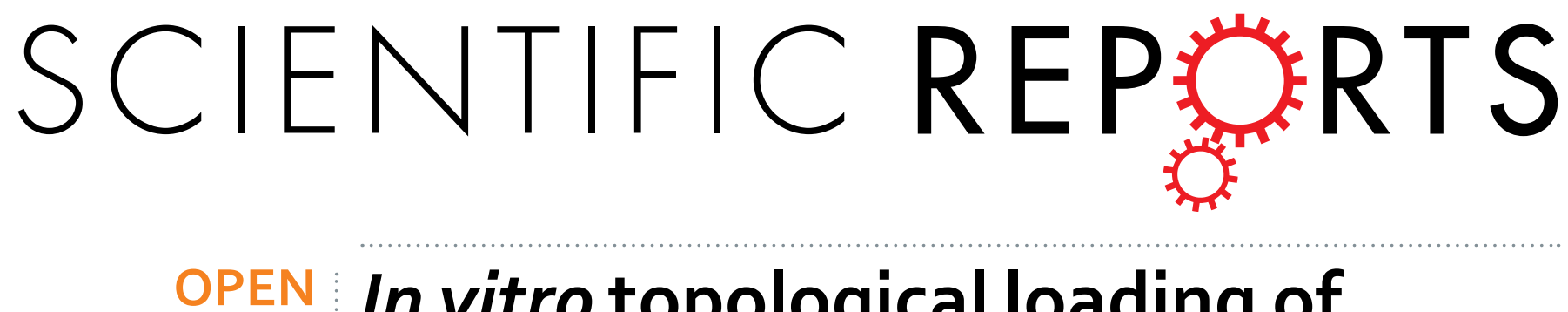

\title{
In vitro topological loading of bacterial condensin MukB on DNA, preferentially single-stranded DNA \\ rather than double-stranded DNA
}

Received: 21 April 2016

Accepted: 20 June 2016

Published: 08 July 2016

\author{
Hironori Niki ${ }^{1,2} \&$ KoichiYano $^{1}$
}

Condensin is the major driving force in the segregation of daughter chromosomes in prokaryotes. Core subunits of condensin belong to the SMC protein family, whose members are characterized by a unique ATPase activity and dimers with a V-shaped structure. The V-shaped dimers might close between head domains, forming a ring structure that can encircle DNA. Indeed, cohesin, which is a subfamily of SMC proteins, encircles double-stranded DNA to hold sister chromatids in eukaryotes. However, the question of whether or not condensin encircles the chromosomal DNA remains highly controversial. Here we report that MukB binds topologically to DNA in vitro, and this binding is preferentially singlestranded DNA (ssDNA) rather than double-stranded DNA. The binding of MukB to ssDNA does not require ATP. In fact, thermal energy enhances the binding. The non-SMC subunits MukF and MukE did stimulate the topological binding of MukB, although they hindered DNA-binding of MukB. Recent reports on the distribution of condensin in genomes reveal that actively transcribed genes in yeast and humans are enriched in condensin. In consideration of all these results, we propose that the binding specificity of condensin to chromosome is provided not by the DNA sequence but by the DNA structure, which is ssDNA.

Condensin is an important protein complex for the compaction of chromosomal DNA in organisms ranging from bacteria to humans ${ }^{1-3}$. Prokaryotic condensin consists of a homodimer of an SMC core unit and two types of non-SMC subunits. The SMC homodimer binds to double-stranded DNA, and to ssDNA ${ }^{4,5}$. The MukBEF complex is a bacterial condensin complex in Eshcrichia coli and related bacteria ${ }^{6}$. MukB is the SMC protein, and MukE and MukF are non-SMC subunits. MukF, which is a member of the kleisin protein family ${ }^{7}$, forms a stable complex with MukE in vivo ${ }^{8}$. In living cells that encode MukB-GFP, a portion of the MukB molecules are distributed to discrete foci in the vicinity of the origin of replication in collaboration with MukE and MukF ${ }^{9,10}$. Thus the MukBEF complexes are formed at specific sites on a bacterial chromosome, and then each of the MukBEF-DNA complexes assembles to pack chromosomal DNA into a nucleoid. However, the mechanism by which particular chromosome sites are chosen for loading of the MukBEF complex remains unclear. Strangely, an in vitro analysis indicated that the DNA-binding of MukB is remarkably inhibited by non-SMC subunits of MukE and MukF ${ }^{8}$. In an earlier study, a globular domain of MukB was shown to have DNA-binding ability ${ }^{11}$. And MukF is known to interact with the globular domains of $\mathrm{MukB}^{12}$, suggesting that MukF covers the DNA-binding domain of MukB. To address this conflict between the in vivo and in vitro results regarding the DNA-binding of MukB, we considered that a ring structure of MukB dimers holds DNA strands as seen in a cohesion ring. Although previous reports have suggested that condensin physically encircles DNA in an isolated condensin-DNA complex ${ }^{13,14}$, it remains a matter of controversy whether condensin binds topologically. In the case of cohesin, the topological binding has recently been established by a topological loading assay for purified yeast cohesion, which is developed by Murayama and Ullmann (2014) (MU assay hereinafter) $)^{15,16}$. In the present study, we investigated the topological binding of bacterial condensin to DNA by using the MU assay with some modifications.

${ }^{1}$ Microbial Genetics Laboratory, Genetic Strains Research Center, National Institute of Genetics, 1111, Yata, Mishima, Shizuoka, 411-8540 Japan. ${ }^{2}$ Department of Genetics, SOKENDAI (The Graduate University for Advanced Studies) 1111, Yata, Mishima, Shizuoka, 411-8540 Japan. Correspondence and requests for materials should be addressed to H.N. (email: hniki@nig.ac.jp) 


\begin{abstract}
Results
Assay for in vitro topological loading of MukB on DNA. To test the topological loading of MukB on DNA, the MU assay was applied for purified MukB proteins. The hexa-histidine-tagged proteins of each of MukB, MukE, and MukF were considered biologically functional because they could complement their deletion phenotypes (data not shown), and then each protein was purified (Supplementary Fig. S1). Purified histidine-tagged MukB was incubated with a variety of DNA substrates under a condition of low salt $(25 \mathrm{mM} \mathrm{KCl})$, and then histidine-tagged MukB was recovered by affinity beads to a histidine-tag after washing with high salt buffer $(750 \mathrm{mM} \mathrm{KCl})$ to eliminate the salt-sensitive binding of MukB to DNA. DNA substrates that were pulled down with histidine-tagged MukB were analyzed by agarose gel electrophoresis. Linear double-stranded DNA (ldsDNA) was not retrieved, but both covalent closed circular DNA (cccDNA), which is dsDNA, and cssDNA were retrieved with histidine-tagged MukB (Fig la). The efficiency of DNA retrieval was $4.6 \%$ for cccDNA, and it was $10.6 \%$ for cssDNA (Fig $1 \mathrm{~b}$ ). The retrieval of circular DNA was significantly higher than the $0.6 \%$ retrieval observed for ldsDNA. MukB continuously preferred to bind to cssDNA rather than to other DNA substrates, although the ratios of retrieved DNA varied in each lot of the purified protein (Supplementary Fig. S2). Thus, MukB has the ability to bind to DNA in a salt-resistant manner. This binding is dependent on the topology of DNA substrates, suggesting that MukB topologically loads on DNA.
\end{abstract}

Preferential loading of MukB to cssDNA. To confirm a preference for DNA-binding to circular DNA, we tested the binding kinetics of MukB to cccDNA and cssDNA, respectively. When cssDNA was used as the substrate, a higher yield of retrieved DNA was obtained (Fig 1c). Thus the MU assays indicate that MukB has a DNA-binding activity that is resistant to high salt, and it prefers cssDNA to ldsDNA and cccDNA for DNA-binding.

It was uncertain whether MukB preferentially bound to ssDNA independent of the DNA topology. We tested the binding of MukB to linear single-stranded DNA (lssDNA). To prepare lssDNA, cssDNA of pUC119 was hybridized with a DNA oligomer including the PstI site, and then cut by the restriction enzyme PstI. All of the hybridized cssDNAs were almost certainly linearized because they were sensitive to exonuclease $\mathrm{T}$, which is a ssDNA-specific nuclease (Fig. 2a). No lssDNA was retrieved by the MU assay (Fig. 2a). In addition, we used heat-denatured DNAs for the MU assay. Heating of ldsDNA produces lssDNA, and heating of open circular DNA (ocDNA) produces both lssDNA and cssDNA. Heat-treated ocDNA became a more effective substrate for DNA-binding of MukB, but heat-treated ldsDNA did not (Fig. 2c). Thus, efficient retrieval of the DNA substrate by using the MU assay was required for not only ssDNA, but also DNA topology. These results indicate that MukB holds onto DNA within its ring structure-i.e., topological binding occurs in the case of cohesin.

Effect of SSB on the topological binding of MukB. In vivo, single-stranded-binding proteins (SSBs) protect ssDNA from attack by DNA-damage agents or remove the secondary structure of DNA. In E. coli, tetramers of SSB are strongly bound to ssDNA of the chromosome so that other DNA-binding proteins cannot access the ssDNA segment covered by SSB. We examined the effects of SSB on the binding of MukB to cssDNA. As the concentration of SSB was increased, the mobility of cssDNA was proportionally shifted in an agarose gel and the saturation binding of SSB was $2.0 \mu \mathrm{g}$ in a reaction mixture (Fig. 3a). The saturation binding of SSB to cssDNA markedly inhibited the topological binding of MukB to cssDNA (Fig. 3b). Pre-mixing of cssDNA and MukB on ice did not improve the efficiencies of cssDNA retrieval by MukB. These results suggest that a direct interaction of MukB with ssDNA might be essential for topological binding of MukB to cssDNA.

Release of MukB from cssDNA by linearization. When the ring of SMC dimers entraps circular DNA, linearization of the circular DNA causes the entrapped DNA to slide out from the SMC ring ${ }^{15}$. To linearize cssDNA that was bound by MukB, cssDNA was previously hybridized with oligomers that included a recognition site by a restriction enzyme. In the MU assay using hybridized cssDNA, the retrieved DNA on beads was digested by PstI (Fig. 3d) or both PstI and BamHI (Fig. 3f). A large amount of cssDNA was released into the supernatant of a DNA-loading reaction after digestion, although a small amount of cssDNA was recovered in the supernatant without digestion. Even though cssDNA was completely digested by both PstI and BamHI, a large amount of cssDNA remained on the beads (Fig. 3f). It was likely that the secondary structure of ssDNA hindered the release of digested DNA from the ring of MukB. To improve the release of DNA from the MukB ring, the secondary structure of ssDNA was removed by SSB. Addition of a moderate amount of SSB $(0.5 \mu \mathrm{g})$ enhanced the release of the digested DNA from the MukB immobilized on beads, as expected (Fig. 3e,g). These results support the notion that the MukB condensin entraps ssDNA.

Effect of MukE and MukF on topological binding of MukB. To confirm the effect of non-SMC subunits in the topological binding of MukB, purified MukE and MukF were added to the reaction mixture of MukB and cssDNA. The stoichiometry of the MukBEF complex in vivo is estimated at $\mathrm{B}_{4} \mathrm{E}_{4} \mathrm{~F}_{2}$ or $\mathrm{B}_{2} \mathrm{E}_{4} \mathrm{~F}_{2}$, based on the quantitative analyses of fluorescent-labeled Muk proteins in a living cell ${ }^{10}$. On the other hand, biochemical analysis has suggested that the stoichiometry of active MukBEF is $\mathrm{B}_{2} \mathrm{E}_{2} \mathrm{~F}_{1}$, and the stoichiometry of the inactive form that does not bind DNA is $\mathrm{B}_{2} \mathrm{E}_{4} \mathrm{~F}_{2}{ }^{8}$. In the present study, MukE and MukF did not deteriorate topological binding of MukB when excess amounts of MukE and MukF $\left(\mathrm{B}_{2} \mathrm{E}_{8} \mathrm{~F}_{8}\right)$ were added to the reaction mixtures for the MU assay (Fig. 4a,b). On the contrary, the topological binding was significantly improved by the reaction with a higher concentration of proteins (Fig. 4c). The effect of MukE and MukF on topological binding of MukB is notable because these proteins deteriorate DNA-binding activity of $\mathrm{MukB}^{8}$. This result suggests that there are at least two modes of DNA binding of MukB to DNA in vitro.

When the cssDNA retrieved from the reaction with MukE and MukF was analyzed by agarose gel electrophoresis using the fluorescent dye SYBRGreenII (SGII), additional bands appeared below the band of cssDNA 
a
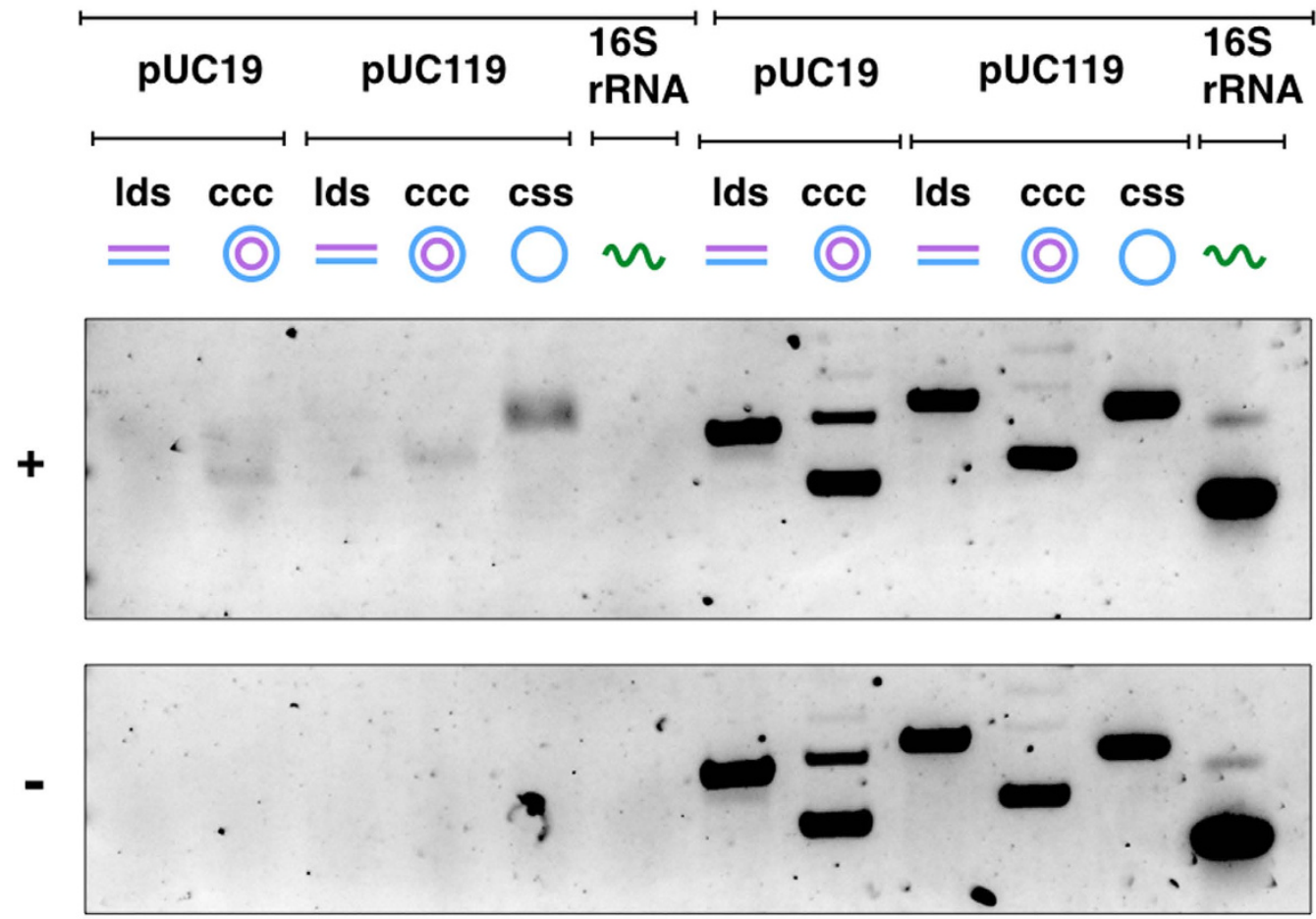

b

C
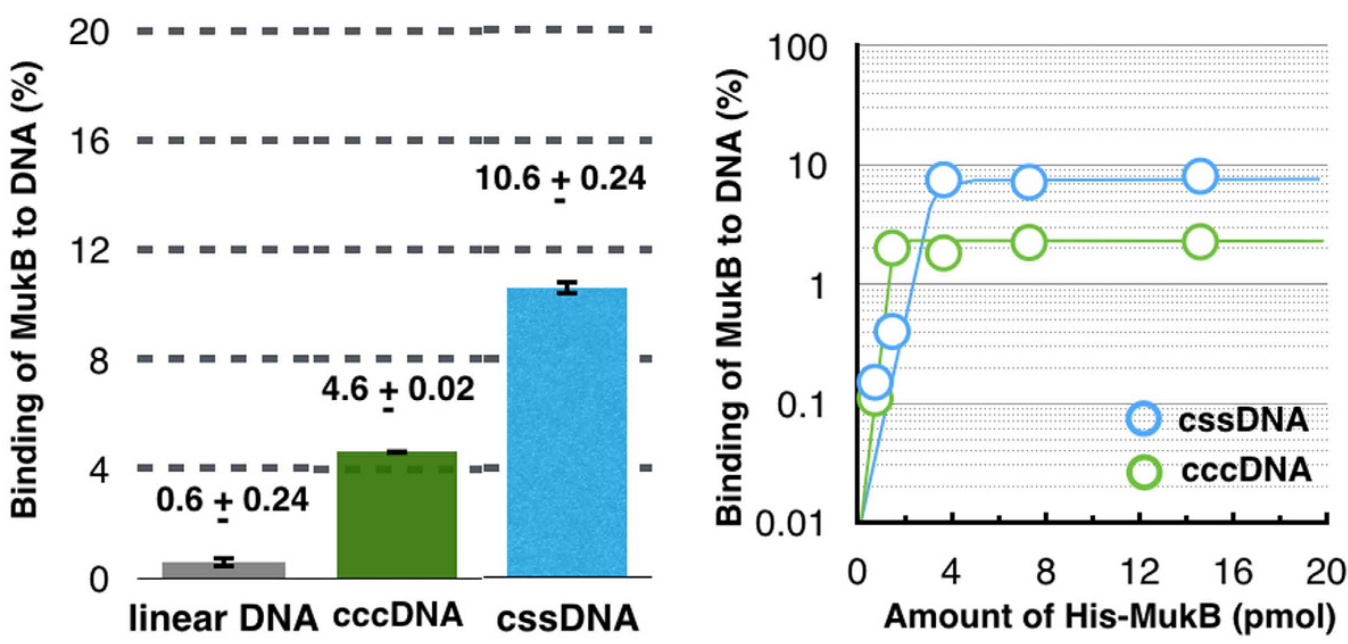

Figure 1. In vitro loading of MukB onto DNA. (a) Agarose gel electrophoresis of retrieved DNA from the MU assay. Various types of DNA and $16 \mathrm{~S}$ rRNA were tested for the MU assay with histidine-tagged MukB $(+)$ and without it (-). (b) Quantification of retrieved DNA by the MU assay. The means and standard deviation were calculated from two independent experiments. (c) Kinetics of histidine-tagged MukB loading onto cssDNA (blue) and cccDNA (green). Various amounts of histidine-tagged MukB were tested with pUC119 DNA (100 ng).

(Fig. 4a). These bands were broader than the band of cssDNA. The bands appeared in lanes on which MukF alone was loaded (Fig. 4d). Indeed, the emission of light by SGII was detected in a solution when MukF was mixed with SGII (Supplementary Fig. S3a). The band was sensitive to proteinase K, but not to nucleases. A profile of the absorbance spectrum showed that MukF had strong absorbance at $280 \mathrm{~nm}$, but not $260 \mathrm{~nm}$, which is the wavelength for the maximum absorbance of nucleic acids (Supplementary Fig. S3b). These results indicate that the MukF protein itself was detected in the agarose gel by SGII. This ability of MukF to bind to and activate SGII warrants further analysis in future studies. 

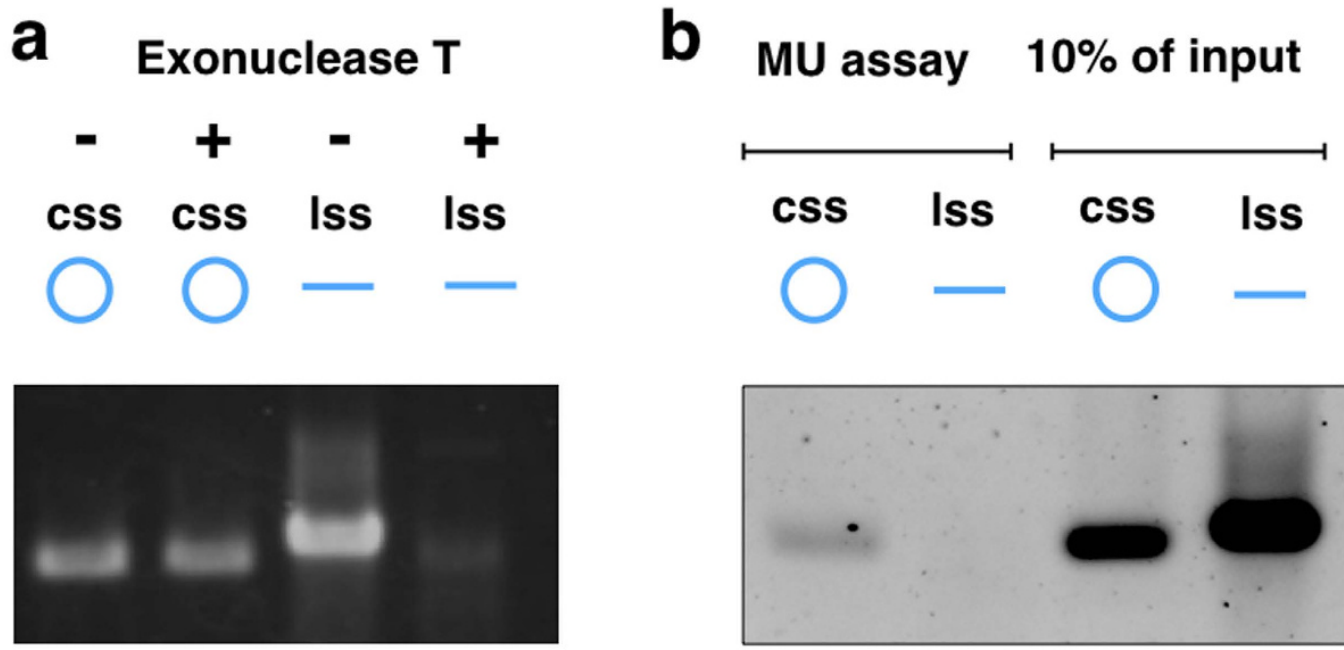

MU assay
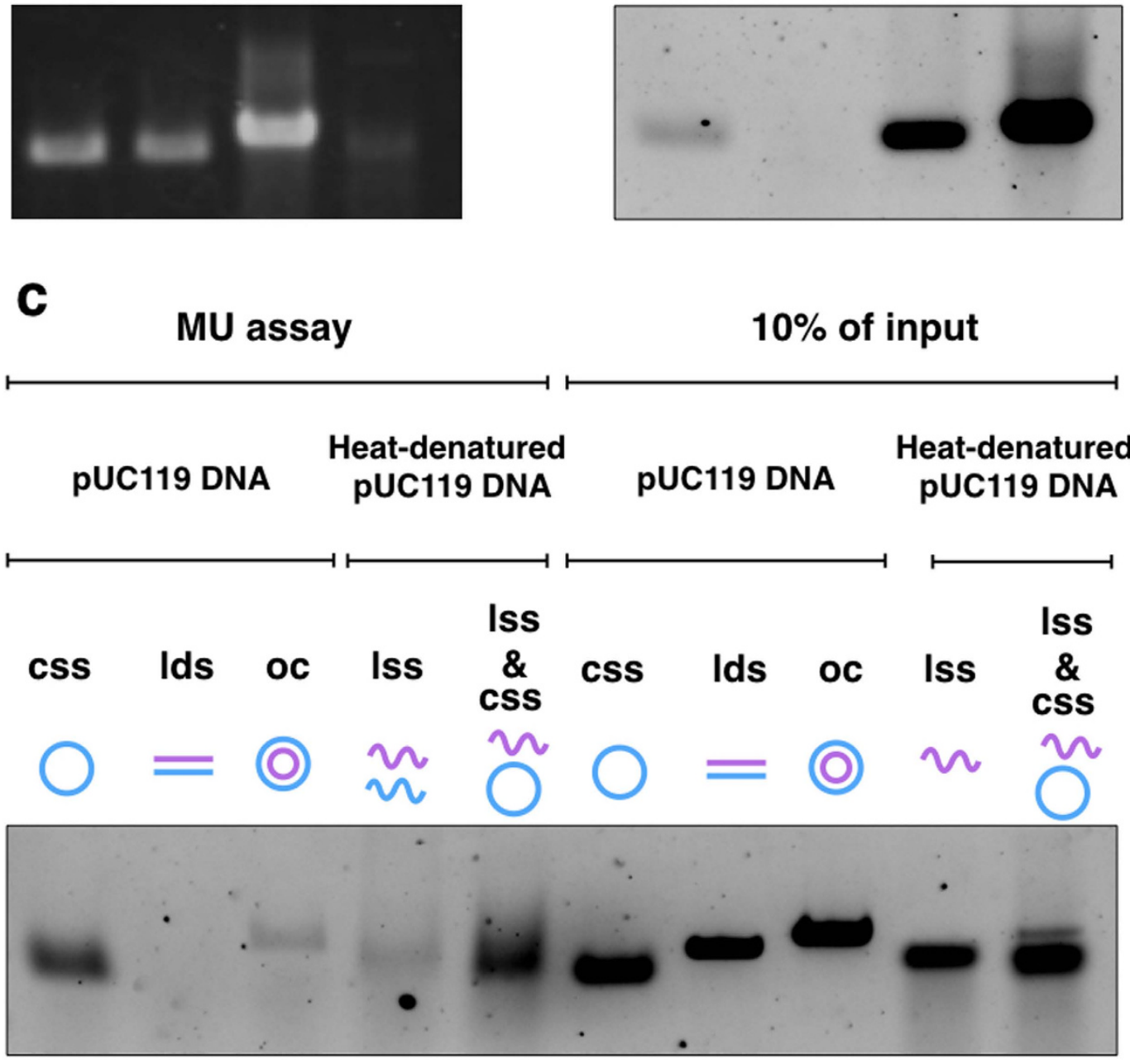

Figure 2. DNA Topology and single strand-mediated DNA-binding of MukB. (a) Agarose gel electrophoresis of ssDNA. Exonuclease T completely digested lssDNA of pUC119 (184 ng), but not cssDNA (100 ng). The gel was stained by ethidium bromide. (b) Agarose gel electrophoresis of retrieved ssDNA from the MU assay. The assay used $100 \mathrm{ng}$ of ssDNA in each reaction. (c) Agarose gel electrophoresis of the MU assay using heatdenatured DNA. ldsDNA and ocDNA were boiled at $95^{\circ} \mathrm{C}$ for $5 \mathrm{~min}$, and then quickly chilled.

ATP is not required for topological binding of MukB. The MU assays above were carried out without ATP, indicating that topological binding of MukB to cssDNA does not require ATP. MukB is the ABC type of ATPase. Its ATPase activity is essential for physiological functions in E. coli. We tested the effect of ATP on binding of MukB to DNA. When $2 \mathrm{mM}$ ATP was added to the reaction mixture, the amount of cssDNA retrieved was not significantly higher than in the reaction without ATP (Fig. 4e). To confirm the requirement of ATP hydrolysis, a mutant form of MukB was used for the reaction. The $\mathrm{MukB}^{\mathrm{K} 40 \mathrm{~A}}$ protein has a mutation in an active center of ATP hydrolysis and cannot complement the deficiency of the MukB deletion mutation. However, efficient typological binding of $\mathrm{MukB}^{\mathrm{K} 40 \mathrm{~A}}$ was observed (Fig. 4f). In addition, when $14.6 \mathrm{pmol}$ of MukB with MukE and MukF $\left(\mathrm{B}_{2} \mathrm{E}_{8} \mathrm{~F}_{8}\right)$ was incubated with cssDNA, ATP addition did not stimulate the topological binding of MukB (Fig. 4g). Thus, energy transfer from ATP is not necessary for topological binding of the MukB to cssDNA. These results are consistent with previous reports that ATP hydrolysis enhances the dissociation of MukBEF from DNA, but not the association of MukBEF to DNA ${ }^{10,17}$. Instead, the temperature after mixing cssDNA with MukB is critical 
a

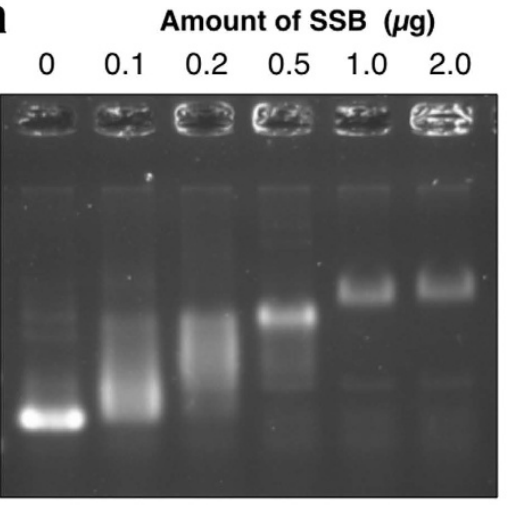

b

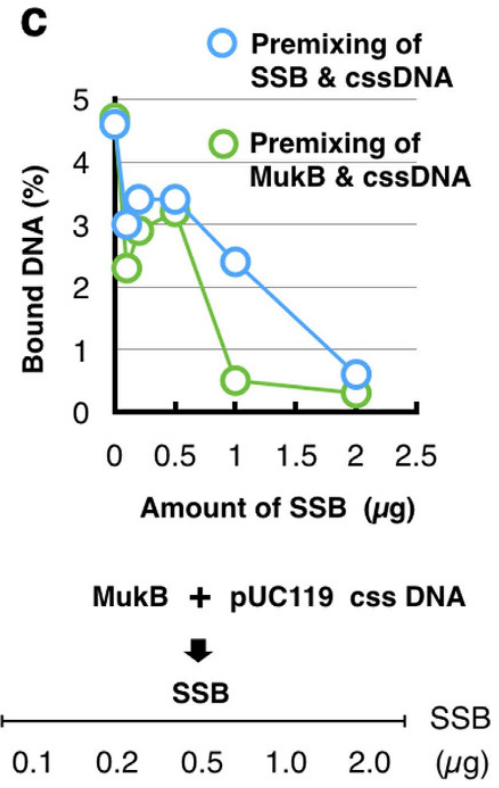

d<smiles>c1ccccc1</smiles>

css DNA of pUC119 hybridized with the Pstl oligomer
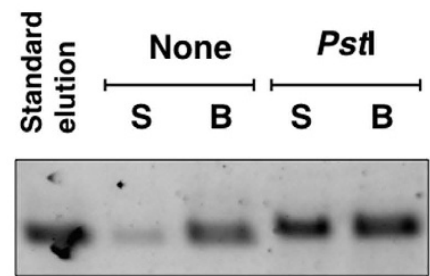

f
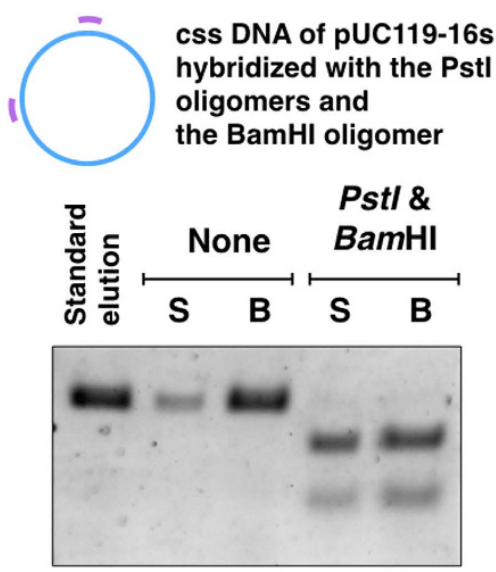

$\mathbf{e}$
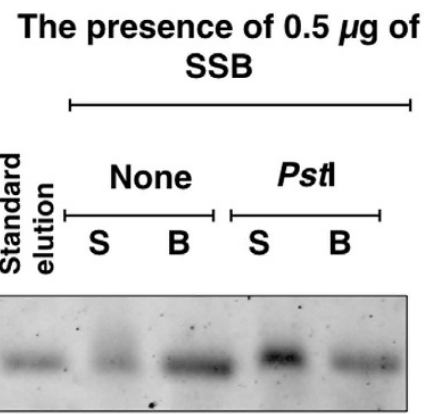

g

The presence of $0.5 \mu \mathrm{g}$ of SSB

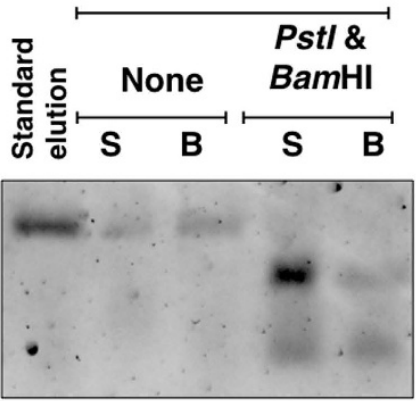

Figure 3. SSB and Topological binding of MukB. (a) Agarose gel electrophoresis of gel mobility shift assay. Various amounts of E. coli SSB were bound to the cssDNA of pUC119 (100 ng) after incubation in HKD buffer on ice for $10 \mathrm{~min}$. (b) Agarose gel electrophoresis of the MU assay in the presence of SSB. Pre-incubation of SSB and cssDNA was carried out as follows. A mixture of SSB and cssDNA was incubated on ice for $10 \mathrm{~min}$, and then histidine-tagged MukB ( $7.3 \mathrm{pmol})$ was added. Next, the mixture was incubated on ice for $5 \mathrm{~min}$, and then incubated at $37^{\circ} \mathrm{C}$ for $10 \mathrm{~min}$ before the $\mathrm{MU}$ assay. On the other hand, pre-incubation of histidine-tagged MukB 
and cssDNA was carried out as follows. A mixture of MukB and cssDNA was incubated on ice for $10 \mathrm{~min}$, and then SSB was added. Next, the mixture was incubated on ice for $5 \mathrm{~min}$, and then it was incubated at $37^{\circ} \mathrm{C}$ for $10 \mathrm{~min}$ before the MU assay. (c) Quantification of retrieved DNA from the MU assay in the presence of SSB. Premixing of SSB and cssDNA is indicated in blue circles, and premixing of MukB and cssDNA is indicated in green circles. (d) The cssDNA hybridized with the oligomer including a PstI site was analyzed by the MU assay. After washing the immobilized MukB-DNA complexes on beads, they were digested with the restriction enzyme PstI, and the reaction mixture was separated into a supernatant fraction (S) and a bead fraction (B). (e) SSB $(0.5 \mu \mathrm{g})$ was added to the digestion reaction. (f) The cssDNA hybridized with the oligomers including the PstI site or the BamHI site was analyzed by the MU assay. The immobilized MukB-DNA complex on beads was digested with the restriction enzymes PstI and BamHI. (g) SSB $(0.5 \mu \mathrm{g})$ was added to the digestion reaction.

for the efficient binding of MukB. The binding reaction was not boosted on ice, but was quickly enhanced at $37^{\circ} \mathrm{C}$ (Fig. 4h). Thus thermal motion is an important promoter of the formation of the topologically bound MukB-DNA complex.

\section{Discussion}

We identified topological loading of MukB to circular DNA, although the ratio of topological binding on DNA was low (Fig. 1b). In the case of the fission yeast cohesin, about 5\% of input DNA was retrieved in the presence of cohesin alone, although the Mis4-Ssl3 cohesin loader improved the amount of retrieved DNA to about 25\%, depending on the presence of $\mathrm{ATP}^{15}$. It has been considered that there is no loader for the MukB condensin in E. coli, based on the phenotypes of gene deletion mutants in a gene knockout library of E. coli $^{18}$.

Although the suggested preference for ssDNA over dsDNA is apparent in Fig. 1b,c, it should be noted that this preference is not so pronounced at lower concentrations of purified MukB. Thus, we cannot exclude the possibility that MukB exhibits significant loading onto both dsDNA and ssDNA in vivo. However, the stable ssDNA segment that is produced by the opening of dsDNA is not ubiquitous on the bacterial genome, or rather it is restricted as chromosomal segments that were highly transcribed in the manner of rRNA and tRNA genes. Therefore, such restriction would contribute to the specific localization of the MukBEF complex in the vicinity of replication origin, as discussed below.

In contrast to the proportional binding of SSB to cssDNA, the ratio of retrieved cssDNA did not decrease in proportion to the amount of SSB (Fig. 3c). A moderate amount of SSB $(0.2-0.5 \mu \mathrm{g})$ did not interfere with the binding of histidine-tagged MukB to cssDNA. Because SSB removes the secondary structure of cssDNA, cssDNA may be a better substrate for topological binding of MukB despite the masking of cssDNA by SSB. In vivo, free ssDNA is rapidly bound by SSB to protect the DNA region. As a matter of course, the complete covering by SSB inhibits access to the ssDNA. If moderate binding of SSB occurs only at a specific region on a bacterial chromosome, this would be helpful for the localization of MukB in vivo.

The condensin complexes that topologically bind to DNA were isolated from Saccharomyces cerevisiae and Bacillus subtilis ${ }^{13,14}$. Proteolytic cleavage of non-SMC subunits causes the release of DNA from the isolated complexes, indicating that the ring structure composed of the SMC core unit and non-SMC subunits is responsible for the topological engagement of DNA. By contrast, our reconstitution experiments of the MukB-DNA complex show that MukB alone is sufficient for the topological binding to DNA. We cannot exclude the possibility that this binding mode of MukB is specific in vitro. Alternatively, it is conceivable that the topological binding is the first step toward formation of the condensin-DNA complex, after which a more stable complex would be formed with non-SMC subunits. Interestingly, E. coli RecN, which is a highly conserved, DNA damage-inducible SMC protein, wraps DNA by forming a polymer via head-head engagement ${ }^{19}$. A similar binding mode of MukB could be included in the processes of condensin complex formation.

$\mathrm{MukB}^{\mathrm{K} 40 \mathrm{~A}}$ could bind to DNA in vitro topologically (Fig. 4f). In contrast, many MukB mutants of ATPase activity have been analyzed and found to be incapable of binding to DNA in vivo ${ }^{10,20}$. However, the ATPase activity of MukB is not directly involved in DNA binding, but rather is involved in coordinating MukB interactions with non-SMA subunits and DNA ${ }^{12,17}$. In addition, the DNA binding of RecN is not dependent on its ATPase activity, because the ATPase-deficient $\mathrm{RecN}^{\mathrm{K} 35 \mathrm{~A}}$ mutant can assemble at a damaged site on the chromosome ${ }^{21}$. Once $\mathrm{RecN}^{\mathrm{K} 35 \mathrm{~A}}$ assembles, however, disassembly of $\mathrm{RecN}^{\mathrm{K} 35 \mathrm{~A}}$ does not occur after the release of DNA damage stress, and thus the cell growth is inhibited. Therefore, it is likely that ATPase activity is not necessary for the binding of bacterial SMC proteins to DNA. Instead, ATPase activity would contribute to the turnover of assembled SMC proteins on DNA.

Electron microscopic observation has revealed two forms of the purified MukB: the open form is a V-shaped dimer, and the closed form is a filamentous dimer ${ }^{22,23}$. We hypothesize that the arms of MukB dimers are flexible and change between the open form or closed form depending on the thermal fluctuation (Fig. 4i). Occasionally the open form captures DNA, and then changes into the closed form. After the MukB dimer captures ssDNA, the closed form would become static because a part of the inner interface of a MukB globular domain interacts with ssDNA. Thus MukB would keep the captured DNA steady inside the ring of the dimer. Further, the MukB-DNA complex might be strengthened by MukEF, and then each of the MukBEF-DNA complexes would be assembled to compact chromosomal DNA. We infer that ATP hydrolysis is required for dissociation of the MukBEF-DNA complex from the assembled complexes.

As is usual with in vitro analysis, the biochemical properties of purified Muk proteins do not always reflect physiological functions faithfully. Even if the topological binding of MukB to DNA is functional in vivo, it is unclear whether the in vivo substrate is ssDNA or dsDNA. Nevertheless, our results motivate us to consider the topological loading of MukB to ssDNA in vivo because of an understanding about the restricted loading of 


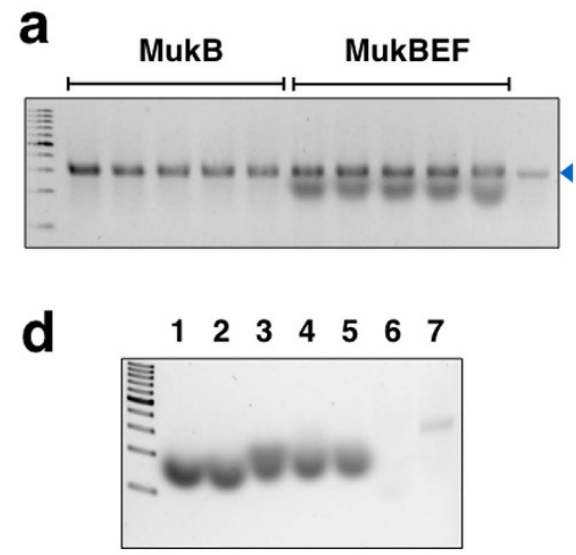

e $7.3 \mathrm{pmol}$ His-MukB

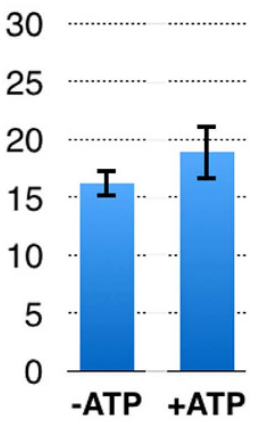

f

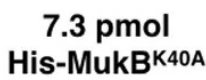

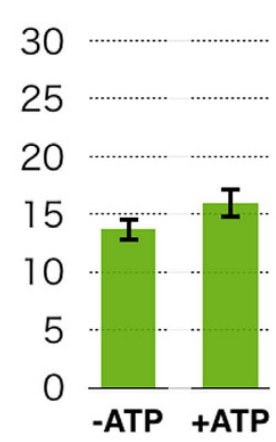

b

\author{
$7.3 \mathrm{pmol}$ \\ His-MukB
}

MukB: $E: F=2: 8: 8$
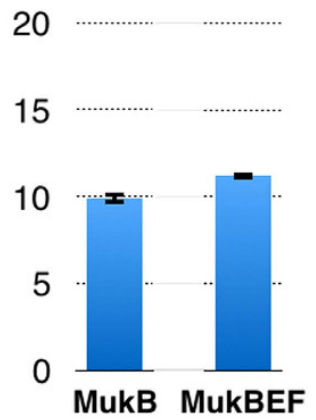

g

\section{6 pmol \\ His-MukB}

MukB: $E: F=2: 8: 8$

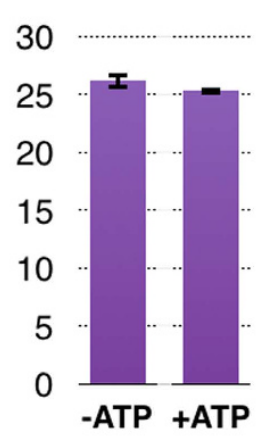

C

14.6 pmol

His-MukB

\section{MukB: $E: F=2: 8: 8$}

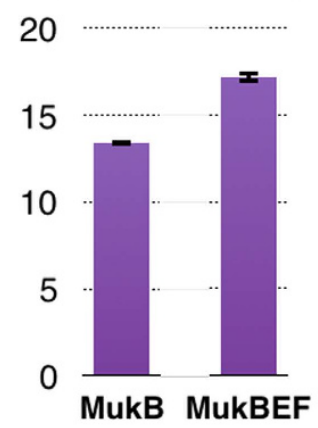

h

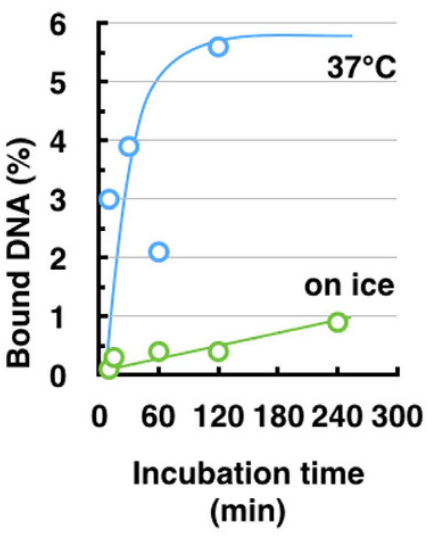

i

MukB dimer

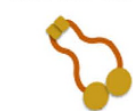

Closed form

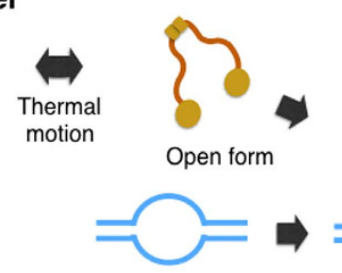

Melting of dsDNA

$$
\text { . }
$$

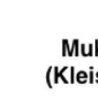

$\underset{\text { MukF }}{\text { (Kleisin) }}$

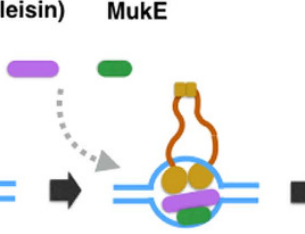

Formation of

MukBEF on ssDNA
Assembly of MukBEF on ssDNA to compact chromosome

Figure 4. Topological DNA-binding of histidine-tagged MukB in the presence of MukEF and ATP. (a) Agarose gel electrophoresis of the MU assay. Five reactions with MukB or MukBEF were analyzed and detected by fluorescence of SYBRGreen II. The arrowhead indicates cssDNA of pUC119. (b) Effect of non-SMC subunits, MukE and MukF on retrieved DNA from the MU assay; MukB (7.3 pmol), MukE (29.2 pmol), MukF (29.2 pmol), and pUC119 cssDNA (100 ng). Means and standard deviations were calculated from five independent experiments. (c) Effect of non-SMC subunits, MukE and MukF on retrieved DNA from the MU assay; MukB (14.6 pmol), MukE (58.4 pmol), MukF (58.4 pmol), and pUC119 cssDNA (100 ng). Means and standard deviations were calculated from five independent experiments. (d) Agarose gel electrophoresis of MukF alone. Purified histidine-tagged MukF in HKD buffer was loaded in agarose gel electrophoresis and stained by SYBRGreen II. The results are shown for $\mathrm{MukF}$ (30 ng) incubated at $37^{\circ} \mathrm{C}$ overnight (lane 1), MukF without incubation (lane 2), MukF incubated with DNase I at $37^{\circ} \mathrm{C}$ overnight (lane 3), MukF incubated with RNase A at $37^{\circ} \mathrm{C}$ overnight (lane 4 ), MukF incubated with mung bean nuclease at $37^{\circ} \mathrm{C}$ overnight (lane 5), MukF incubated with proteinase $\mathrm{K}$ at $37^{\circ} \mathrm{C}$ overnight (lane 6), and $10 \mathrm{ng}$ of cssDNA of pUC119 (lane 7). (e) Effect of $2 \mathrm{mM}$ ATP on DNA retrieval from the MU assay. (f) Effect of $2 \mathrm{mM}$ ATP on DNA retrieval of MukB ${ }^{\mathrm{K} 40 \mathrm{~A}}$ from the MU assay. (g) Effect of $2 \mathrm{mM}$ ATP on DNA retrieval from the MU assay; MukB (14.6 pmol), MukE (58.4 pmol), MukF (58.4 pmol), and pUC119 cssDNA (100 ng). Means and standard deviations were calculated from five independent experiments. (h) Effect of the reaction temperature on DNA retrieval from the MU assay. Reaction mixtures of MukB (7.3 pmol) and pUC119 cssDNA (100 ng) were incubated at $37^{\circ} \mathrm{C}$ (blue) and on ice (green). The means and standard deviations $(\mathbf{b}, \mathbf{d}, \mathbf{e})$ were calculated from five independent experiments. (i) A model of topological binding of MukB in E. coli cells. 
condensin on chromosome. Recent reports have demonstrated that condensin is specifically localized at ssDNA segments in a cell. The binding of condensin to ssDNA is found in both Schizosaccharomyces pome and Bacillus subtilis $^{5,24}$. Because SMC proteins maintain biochemical activities beyond the three domains of life, the property of topological binding of MukB to ssDNA may also apply to these SMC proteins. From this viewpoint, we could reconsider some of the previous results on the distribution of condensin in genomic DNA. First, the SMC proteins of $B$. subtilis are highly accumulated at rRNA genes and the tRNA genes, which are highly transcribed genes, in addition to the ParB-binding sites that are putative loading sites for condensin ${ }^{25}$. Moreover, rRNA genes of yeast also are rich in condensin ${ }^{26,27}$. In fact, a plasmid containing the rRNA gene has been isolated with the condensin complex from yeast, and this complex is also known to bind the plasmid DNA topologically ${ }^{28}$. Moreover, tRNA genes also are targets for the binding of yeast condensin ${ }^{29}$, and the clustering of tRNA genes that scatter at budding yeast chromosomes is governed by conden $\sin ^{30}$. Finally, two recent reports employing Chip-Seq analyses indicated that condensin is enriched at actively transcribed genes by RNA polymerase II in yeast and humans ${ }^{31,32}$. Condensin accumulates at unwound DNA at the transcription start site. These reports are well consistent with our present results, indicating that the specific loading of MukB to ssDNA can account for the in vivo localization of condensin at actively transcribed genes. It is thought that ssDNA is remarkably transient in vivo, and therefore only highly actively transcribed genes including the rRNA and the tRNA genes might be favorable target sites for topological loading by condensin. Indeed, multiple copies of the rRNA genes in E. coli and B. subtilis are dispersed in the vicinity of the origins of replication. This arrangement of the rRNA genes is suitable for packaging of newly replicated DNA strands in an orderly manner. Moreover, the real copy number of the rRNA genes is greater than that required for sufficient synthesis of rRNA ${ }^{33}$. This fact suggests that the multiple copies of the rRNA genes perform other functions in addition to their role in rRNA synthesis. In conclusion, the condensin-loading sites are determined by DNA states, rather than DNA sequences. Our findings suggest that ssDNA generated by active transcription is a more important site for biological activity than previously thought.

\section{Materials and Methods}

Preparation of substrate DNA. We used purified DNA of pUC19, pUC119, and pUC119-16S as substrates of the MU assay. The plasmid pUC119 is a derivative from pUC19 to produce ssDNA that was packaged into M13 phage particles. The DNA fragments encoding 16S ribosomal RNA were amplified from E. coli MG1655 by using a primer set: rrnB-16S-F(Bam) for ggaggatccaaattgaagagtttgatcatggctc, and rrnB-16S-R(Pst) for ctgctgcagtaaggaggtgatccaaccgc. The amplified DNA fragment was digested by Bam $\mathrm{HI}$ and PstI, and then inserted into the BamHI and PstI sites of pUC119, resulting in pUC119-16S.

To extract ssDNA of pUC119 from M13 phage particles, E. coli MV1184 cells harboring pUC119 were cul-

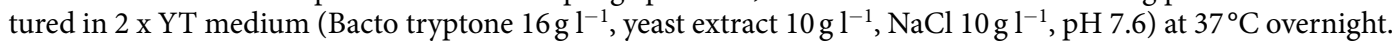
To package the ssDNA of pUC119 into M13 phage particles, the cells were infected with a helper page M13KO7. Phage lysate of M13KO7 was added into $1 \mathrm{ml}$ of overnight culture of MV1184 harboring pUC119 under the condition that the multiplicity of infection (moi) was between 2 and 10 . Infected cells were incubated at $37^{\circ} \mathrm{C}$ for 1 hour with gentle shaking. The culture was placed into $10 \mathrm{ml}$ of $2 \times$ YT medium containing $50 \mu \mathrm{g} \mathrm{ml}^{-1}$ of kanamycin, and then it was incubated until full growth of cells. The culture was centrifuged to remove cells, and then the supernatant was further centrifuged to precipitate phage particles $(100,000 \mathrm{rpm}$ for $30 \mathrm{~min}$ using a TLS-110 rotor; model: Optima TLX, Beckman Coulter Inc.). The precipitate was resuspended in $100 \mu \mathrm{l}$ of TE solution (10 mM Tris-HCl: pH 8.0, 0.1 mM EDTA) containing RNase A $\left(5 \mu \mathrm{g} \mathrm{ml}^{-1}\right)$ and DNase I $\left(10 \mu \mathrm{g} \mathrm{ml}^{-1}\right)$, and the suspension was incubated at $37^{\circ} \mathrm{C}$ for $30 \mathrm{~min}$. The pUC119 cssDNA was extracted with a saturated solution of phenol/chloroform. Finally, the extracted DNA was precipitated by ethanol precipitation and resuspended in TE buffer.

The pUC19 and 119 cccDNA were extracted from E. coli cells by using silica-membrane technology and further purified by using CsCl density-gradient ultracentrifugation (rotor: TLS-110; model: Optima TLX, Beckman Coulter Inc.). ocDNA was purified by density-gradient ultracentrifugation from a DNA solution of pUC119 that was kept at $4{ }^{\circ} \mathrm{C}$ in long-term storage as a laboratory stock.

lssDNA was made by cutting of cssDNA of pUC119. An oligomer including the PstI site (\#881; agtcgacctgcaggcatgca) was hybridized with cssDNA of pUC119 to generate a dsDNA segment. The mixture of the oligomer and pUC119 cssDNA in HKD buffer (25 mM HEPES-KOH; pH7.6, $25 \mathrm{mM} \mathrm{KCl,} 1 \mathrm{mM} \mathrm{DTT}$ ) was heated at $65^{\circ} \mathrm{C}$ for $10 \mathrm{~min}$ and gradually cooled down until room temperature. The hybridized DNA was digested by Pst I, and then purified by phenol/chloroform extraction. To test linearization of pUC119 cssDNA, digested DNA was analyzed by ExoT nuclease.

Ribosomal RNA was extracted form of E. coli cells by Phenol extraction. To isolate 16S RNA, crude RNA extract was centrifuged by $15-60 \%$ glycerol density gradient centrifugation (rotor: TLS-55; model: Optima TLX, Beckman Coulter Inc.).

E. coli strains and plasmid DNA were obtained from National Bioresource Center; E. coli, Mishima, Japan.

Expression and purification of the Muk proteins. Each of the DNA fragments containing the $m u k B$, the $m u k E$, and the $m u k F$ gene was amplified by polymerase chain reaction (PCR) and cloned into an expression vector, pET-28a. The primers used for the PCR were as follows: a set of primers for mukB (mukB-F[Nde]; atcatcatatgattgaacgcggtaaatttcg, mukB-R[EcoBam]; GGAGGATCCGAATTCgctgccgccttaattttaact), a set of primers for mukE (mukE-F[Nde]; atcatcatatgtcatcgacaaatattgaacaag, mukE-R[Bam]; GGAGGATCCttattcttcctctccgctatc), and a set of primers for $m u k F$ (mukF-F; agtgaattttcccagacagtc, mukF-R[Eag]; TTTCGGCCGaatatttgtcgatgacatgcgc). The capital letters in the upper primer sequences indicate the DNA sequences that were inside each of the coding genes. The amplified DNA fragments including the $m u k B$, and the $m u k E$ gene are inserted into the NdeI and the BamHI site of pET-28a. Each of gene products tagged with 6 residues of histidines at their $\mathrm{N}$-terminuses was able to suppress growth deficiency of the $m u k B$ and the $m u k E$ deletion mutant, respectively. On the other hand, the MukF protein was tagged with 6 residues of histidines at their C-terminuses because the MukF protein tagged with 
histidines at their N-terminus did not suppress a defect of the $m u k F$ deletion mutant. To construct such a $m u k F$ gene, the amplified DNA fragment including the $m u k F$ gene was digested by the NdeI. A protruding 5 ' end of the DNA fragment was converted into a blunt end by using T7 DNA polymerase and further digested by EagI. T4 polynucleotide kinase was used to add a phosphate to the ends of the DNA fragments. The modified DNA fragment was inserted into the NdeI and the EagI site of pET-28a. The MukF tagged with 6 residues of histidines at their C-terminuses was able to suppress a defect of the mukE deletion mutant. All muk genes cloned into pET28 a were thoroughly sequenced to confirm the coding products tagged with (His)6. Each of the plasmids that expressed histidine-tagged Muk proteins was transformed into the host BL21(DE3).

To purify the histidine-tagged Muk proteins, BL21(DE3) harboring pET28a-mukB, pET28a-mukE or pET28a-mukF was inoculated into $20 \mathrm{ml}$ of the $\mathrm{L}$ medium (Bacto tryptone $10 \mathrm{~g} \mathrm{l}^{-1}$, yeast extract $5 \mathrm{~g} \mathrm{l}^{-1}, \mathrm{NaCl}^{2} \mathrm{~g} \mathrm{l}^{-1}$, $\mathrm{pH}$ 7.4) with $15 \mu \mathrm{g} \mathrm{ml}^{-1}$ of kanamycin, and incubated at $37^{\circ} \mathrm{C}$ for overnight. All the overnight cultures were inoculated into $1000 \mathrm{ml}$ of $\mathrm{L}$ medium with $15 \mu \mathrm{g} \mathrm{ml}^{-1}$ of kanamycin and gentle shaking at $37^{\circ} \mathrm{C}$. When the optical density of absorbance $600 \mathrm{~nm}$ against in the culture reached 0.3 , isopropyl $\beta$-D-1-thiogalactopyranoside (IPTG, final concentration: $0.1 \mathrm{mM}$ ) was added to the culture to induce histidine-tagged proteins. After further gentle shaking at $37^{\circ} \mathrm{C}$ for 2 hours, cells in the culture were harvested by centrifugation, and then washed by $\mathrm{HK}$ buffer solution ( $50 \mathrm{mM}$ HEPES-KOH: $\mathrm{pH7} .6,100 \mathrm{mM} \mathrm{KCl}$ ). Precipitated cells were frozen by liquid nitrogen and kept in a freezer at $-80^{\circ} \mathrm{C}$. The deep freezer preserved the frozen cells for at least three months.

To extract the his-tagged Muk proteins, the frozen cells were thawed on ice with $30 \mathrm{ml}$ of the sonication buffer (50 mM HEPES-KOH, pH 7.6, $100 \mathrm{mM} \mathrm{KCl,} 1 \mathrm{mM}$ phenylmethylsulfonyl fluoride). Addition of lysozyme was prohibited because of the increment of contaminated proteins in the final fraction. The cell suspension on an ice bath was sonicated by using a BRNSON SONIFIER 250 (duty cycle, constant; output control, 2.5, 10 second, 15 times). Sonicated cells were centrifuged by $10,000 \mathrm{~g}$ for $10 \mathrm{~min}$ to remove the cell debris. The supernatant was decanted into a glass flask. To precipitate proteins in the supernatant, $8.8 \mathrm{~g}$ of ammonium sulfate with fine powder (30\%) was carefully added to $50 \mathrm{ml}$ of the supernatant fluid. After ammonium sulfate was dissolved thoroughly, the solution was stood on ice for $15 \mathrm{~min}$. The mixture was then centrifuged $\left(9,100 \mathrm{~g}, 10 \mathrm{~min}, 4^{\circ} \mathrm{C}\right)$. For purification of the his-tagged MukE protein, ammonium sulfate precipitation at $50 \%$ salt saturation followed by $30 \%$ salt saturation; $6.3 \mathrm{~g}$ of ammonium sulfate was dissolved into the supernatant of ammonium sulfate precipitation at $30 \%$ salt saturation. After standing on ice for $15 \mathrm{~min}$, the mixture was centrifuged again $\left(9,100 \mathrm{~g}, 10 \mathrm{~min}, 4^{\circ} \mathrm{C}\right)$.

The supernatant of ammonium sulfate precipitation was discarded and the precipitate was resuspended in $10 \mathrm{ml}$ of equilibrium buffer ( $50 \mathrm{mM}$ HEPES-KOH, pH 7.6, $100 \mathrm{mM} \mathrm{KCl}, 20 \mathrm{mM}$ imidazole). To remove insoluble particles, the resuspended solution was further centrifuged at $9,100 \mathrm{~g}$ for $10 \mathrm{~min}$. The resuspended solution was then applied into an open column containing $8 \mathrm{ml}$ of TALON metal affinity resin (Clontech Laboratories Inc.) that was equilibrated against the equilibration buffer. The resin was washed with $15 \mathrm{ml}$ of the wash buffer $(30 \mathrm{mM}$ HEPES-KOH, pH 7.6, $750 \mathrm{mM} \mathrm{KCl,} 10 \mathrm{mM}$ imidazole). The histidine-tagged proteins were eluted from the resin by elution buffer ( $50 \mathrm{mM}$ HEPES-KOH, pH 7.6, $100 \mathrm{mM} \mathrm{KCl,} 500 \mathrm{mM}$ imidazole). Each $1 \mathrm{ml}$ of the flow-through fraction was collected into micro-test tubes. To detect eluted protein, the absorbance at $280 \mathrm{~nm}$ in each fraction was measured, and then the peak fractions were pooled.

The fractions containing histidine-tagged protein were loaded onto a Resource Q column ( $1 \mathrm{ml}$; GE Healthcare) that was equilibrated against buffer A (50 mM HEPES-KOH, pH 7.6, $100 \mathrm{mM} \mathrm{KCl).} \mathrm{After} \mathrm{loading} \mathrm{of}$ histidine-tagged protein, the column was washed with $25 \mathrm{ml}$ of buffer A. The column was developed with a linear gradient between $100-1000 \mathrm{mM} \mathrm{KCl}$ by using $20 \mathrm{ml}$ of buffer B ( $50 \mathrm{mM}$ HEPES-KOH, pH 7.6, $1000 \mathrm{mM} \mathrm{KCl}$ ). Each $1 \mathrm{ml}$ of the flow-through fraction was collected into micro-test tubes. Fractions including histidine-tagged protein were dialyzed against buffer C (50 mM HEPES-KOH, pH 7.6, $100 \mathrm{mM} \mathrm{KCl,} 0.1 \mathrm{mM} \mathrm{DTT,} \mathrm{50 \%} \mathrm{(v/v)} \mathrm{glycerol).}$ The concentration of purified protein was measured by a method based on Bradford protein assay (Bio-Rad Protein Assay, Bio-Rad Laboratories). Bovine serum albumin was used as a standard to calculate the protein concentration.

In vitro MukB-loading assay. We used a topological loading assay developed by Murayama and Ullmann $(2014)^{15}$. The method was modified and adapted for our experimental purpose. In general, the loading assay of MukB on DNA was carried out in a $10 \mu \mathrm{l}$ reaction mixture $(25 \mathrm{mM}$ HEPES-KOH, $\mathrm{pH} 7.6,25 \mathrm{mM} \mathrm{KCl}, 1 \mathrm{mM} \mathrm{DTT}$, $1 \mathrm{mM} \mathrm{MgCl}_{2}, 100 \mathrm{ng}$ of substrate DNA, and $7.3 \mathrm{pmol}$ of his-tagged MukB). The reaction mixtures of the MU assay were prepared on ice, and then put into an incubator at $37^{\circ} \mathrm{C}$. After $10 \mathrm{~min}$, the reaction tubes were transferred onto ice and supplemented with $500 \mu \mathrm{l}$ of CP buffer $(25 \mathrm{mM}$ HEPES-KOH, pH 7.5, $500 \mathrm{mM} \mathrm{KCl,} 1 \mathrm{mM}$ DTT, $1 \mathrm{mM} \mathrm{MgCl}_{2}, 5 \%$ glycerol, $0.35 \%$ Triton X-100, $5 \mathrm{mM}$ imidazole). In addition, $10 \mu \mathrm{l}$ of slurry of Dynabeads His-Tag (Novex) that was equilibrated against $\mathrm{CP}$ buffer was added into the reaction tubes and gently mixed. The tubes were then gently rotated at $4^{\circ} \mathrm{C}$ for $30 \mathrm{~min}$. The magnetic beads were washed using CW 1 buffer $(25 \mathrm{mM} \mathrm{HEPES}-\mathrm{KOH}$, $\mathrm{pH} 7.5,750 \mathrm{mM} \mathrm{KCl}, 1 \mathrm{mM}$ DTT, $1 \mathrm{mM} \mathrm{MgCl}_{2}, 0.35 \%$ Triton X-100, $5 \mathrm{mM}$ imidazole) with rotating at $4{ }^{\circ} \mathrm{C}$ for $10 \mathrm{~min}$. The magnetic beads were washed three times using CW1 buffer, followed by one time with CW2 buffer (25 mM HEPES-KOH, pH 7.5, $100 \mathrm{mM} \mathrm{KCl,} 1 \mathrm{mM} \mathrm{DTT,} 1 \mathrm{mM} \mathrm{MgCl} 2,0.1 \%$ Triton X-100, $5 \mathrm{mM}$ imidazole). After the complete removal of CW2 buffer, $10 \mu \mathrm{l}$ of elution buffer $(25 \mathrm{mM}$ HEPES-KOH, pH 7.5, $100 \mathrm{mM} \mathrm{KCl}, 1 \mathrm{mM}$ DTT, $1 \mathrm{mM} \mathrm{MgCl}$, $500 \mathrm{mM}$ imidazole) was added to each reaction tube and incubated at room temperature for $10 \mathrm{~min}$. A supernatant of each reaction was transferred to a new tube and added to $2 \mu \mathrm{l}$ of denature solution $(8 \%$ sodium dodecyl sulfate, $0.1 \mathrm{M}$ EDTA) and $3 \mu \mathrm{l}$ of loading buffer $(0.9 \%$ SDS, $50 \%$ glycerol, $0.05 \%$ bromophenol blue). These samples were analyzed by $0.8 \%$ agarose gel electrophoresis in $1 \mathrm{x}$ TAE buffer $(100 \mathrm{~V}, 30 \mathrm{~min})$. The agarose gels were stained by SYBRGreen II (Takara Bio Inc.). Images of gels were captured by using LuminoGraph (ATTO) or LAS-4000 mini (Fujifilim), and the band intensities were analyzed by using ImageJ 1.48v.

In order to linearize cssDNA that was bound to histidine-tagged MukB, cssDNA that hybridized with oligomers including the PstI site (\#881) was used for the MU assay. For double digestion by Pst I and BamHI, pUC119-16S DNA was cloned the 16S ribosomal DNA fragment into the PstI and BamHI site on the pUC119. 
Oligomers including the PstI and the BamHI site were hybridized with cssDNA of pUC119-16S (PstI \#891; ctccttaCTGCAGgcatgca, BamHI \#896; tacccggGGATCCcaaattga). To digest the retained cssDNA by restriction enzymes, after washing with CW2 buffer, the magnetic beads were resuspended in $10 \mu l$ of digestion buffer ( $25 \mathrm{mM}$ HEPES-KOH, pH 7.5, $750 \mathrm{mM} \mathrm{KCl}, 1 \mathrm{mM} \mathrm{DTT}, 1 \mathrm{mM} \mathrm{MgCl}_{2}$ ) including restriction enzymes, and then incubated for $10^{\circ} \mathrm{C}$ for 1 hour. The digestion buffer was recovered as supernatants (sup), and the magnetic beads were washed with elution buffer as beads fractions.

Gel mobility shift assay. The gel shift assay of cssDNA was carried out in agarose gel electrophoresis with TAE buffer. cssDNA of pUC119 (100 ng) in $\mathrm{HKD}$ buffer including $1 \mathrm{mM} \mathrm{MgCl}{ }_{2}$ was incubated at $4{ }^{\circ} \mathrm{C}$ for $10 \mathrm{~min}$. E .coli SSB was purchased from Bio Academia. The agarose gels were stained by SYBRGreen II (Takara Bio Inc.). Images of gels were captured by using LuminoGraph (ATTO) and band intensities were analyzed by using ImageJ $1.48 \mathrm{v}$.

\section{References}

1. Nasmyth, K. \& Haering, C. H. The structure and function of SMC and kleisin complexes. Annu. Rev. Biochem. 74, 595-648 (2005).

2. Hirano, T. Condensin-Based Chromosome Organization from Bacteria to Vertebrates. Cell 164, 847-857 (2016).

3. Reyes-Lamothe, R., Nicolas, E. \& Sherratt, D. J. Chromosome Replication and Segregation in Bacteria. Annu. Rev. Genet. 46, 121-143 (2012).

4. Niki, H. et al. E.coli MukB protein involved in chromosome partition forms a homodimer with a rod-and-hinge structure having DNA binding and ATP/GTP binding activities. EMBO J 11, 5101-5109 (1992).

5. Hirano, M. \& Hirano, T. ATP-dependent aggregation of single-stranded DNA by a bacterial SMC homodimer. EMBO J 17, 7139-7148 (1998).

6. Niki, H., Jaffé, A., Imamura, R., Ogura, T. \& Hiraga, S. The new gene mukB codes for a 177 kd protein with coiled-coil domains involved in chromosome partitioning of E. coli. EMBO J 10, 183-193 (1991).

7. Fezzie, R. F., Gradia, S. D., Akey, D. \& Berger, J. M. The MukF subunit of Escherichia coli condensin: architecture and functional relationship to kleisins. EMBO J 24, 1921-1930 (2005)

8. Petrushenko, Z. M., Lai, C.-H. \& Rybenkov, V. V. Antagonistic interactions of kleisins and DNA with bacterial Condensin MukB. J Biol Chemi 281, 34208-34217 (2006).

9. Danilova, O., Reyes-Lamothe, R., Pinskaya, M., Sherratt, D. \& Possoz, C. MukB colocalizes with the oriC region and is required for organization of the two Escherichia coli chromosome arms into separate cell halves. Mol Microbiol 65, 1485-1492 (2007).

10. Badrinarayanan, A., Reyes-Lamothe, R., Uphoff, S., Leake, M. C. \& Sherratt, D. J. In vivo architecture and action of bacterial structural maintenance of chromosome proteins. Science 338, 528-531 (2012).

11. Saleh, A. Z. et al. Carboxyl terminal region of the MukB protein in Escherichia coli is essential for DNA binding activity. FEMS Microbiol Lett 143, 211-216 (1996).

12. Woo, J.-S. et al. Structural Studies of a Bacterial Condensin Complex Reveal ATP-Dependent Disruption of Intersubunit Interactions. Cell 136, 85-96 (2009).

13. Cuylen, S., Metz, J. \& Haering, C. H. Condensin structures chromosomal DNA through topological links. Nat Struct Mol Biol 18, 894-901 (2011).

14. Wilhelm, L. et al. SMC condensin entraps chromosomal DNA by an ATP hydrolysis dependent loading mechanism in Bacillus subtilis. Elife 4, 11202 (2015).

15. Murayama, Y. \& Uhlmann, F. Biochemical reconstitution of topological DNA binding by the cohesin ring. Nature 505, 367-371 (2014).

16. Murayama, Y. \& Uhlmann, F. DNA Entry into and Exit out of the Cohesin Ring by an Interlocking Gate Mechanism. Cell 163, 1628-1640 (2015).

17. Cui, Y., Petrushenko, Z. M. \& Rybenkov, V. V. MukB acts as a macromolecular clamp in DNA condensation. Nat Struct Mol Biol 15, 411-418 (2008)

18. Baba, T. et al. Construction of Escherichia coli K-12 in-frame, single-gene knockout mutants: the Keio collection. Mol Syst Biol 2, 2006.0008 (2006).

19. Pellegrino, S. et al. Structural and functional characterization of an SMC-like protein RecN: new insights into double-strand break repair. Structure 20, 2076-2089 (2012).

20. Rybenkov, V. V., Herrera, V., Petrushenko, Z. M. \& Zhao, H. MukBEF, a chromosomal organizer. J. Mol. Microbiol. Biotechnol. 24, 371-383 (2014).

21. Keyamura, K., Sakaguchi, C., Kubota, Y., Niki, H. \& Hishida, T. RecA protein recruits structural maintenance of chromosomes (SMC)-like RecN protein to DNA double-strand breaks. J. Biol. Chem. 288, 29229-29237 (2013).

22. Melby, T. E., Ciampaglio, C. N., Briscoe, G. \& Erickson, H. P. The symmetrical structure of structural maintenance of chromosomes (SMC) and MukB proteins: long, antiparallel coiled coils, folded at a flexible hinge. J Cell Biol 142, 1595-1604 (1998).

23. Matoba, K., Yamazoe, M., Mayanagi, K., Morikawa, K. \& Hiraga, S. Comparison of MukB homodimer versus MukBEF complex molecular architectures by electron microscopy reveals a higher-order multimerization. Biochem. Biophys. Res. Commun. 333, 694-702 (2005)

24. Sutani, T. \& Yanagida, M. DNA renaturation activity of the SMC complex implicated in chromosome condensation. Nature 388, 798-801 (1997).

25. Gruber, S. \& Errington, J. Recruitment of Condensin to Replication Origin Regions by ParB/SpoOJ Promotes Chromosome Segregation in B. subtilis. Cell 137, 685-696 (2009).

26. Freeman, L., Aragon-Alcaide, L. \& Strunnikov, A. The condensin complex governs chromosome condensation and mitotic transmission of rDNA. J Cell Biol 149, 811-824 (2000).

27. Johzuka, K. \& Horiuchi, T. The cis element and factors required for condensin recruitment to chromosomes. Mol Cell 34, 26-35 (2009).

28. Bartosik, A. A. \& Jagura-Burdzy, G. Bacterial chromosome segregation. Acta Biochim. Pol. 52, 1-34 (2005).

29. D’Ambrosio, C. et al. Identification of cis-acting sites for condensin loading onto budding yeast chromosomes. Genes Dev 22, 2215-2227 (2008).

30. Haeusler, R. A., Pratt-Hyatt, M., Good, P. D., Gipson, T. A. \& Engelke, D. R. Clustering of yeast tRNA genes is mediated by specific association of condensin with tRNA gene transcription complexes. Genes Dev 22, 2204-2214 (2008).

31. Sutani, T. et al. Condensin targets and reduces unwound DNA structures associated with transcription in mitotic chromosome condensation. Nat Commun 6, 7815 (2015).

32. Nakazawa, N. et al. RNA pol II transcript abundance controls condensin accumulation at mitotically up-regulated and heat-shockinducible genes in fission yeast. Genes to Cells 20, 481-499 (2015).

33. Yano, K. et al. Multiple rRNA operons are essential for efficient cell growth and sporulation as well as outgrowth in Bacillus subtilis. Microbiol 159, 2225-2236 (2013). 


\section{Acknowledgements}

We are grateful to Y. Murayama for the protocol of the MU assay, and K. Furuya and members of the Microbial Genetic Laboratory for comments on the manuscript. This work was supported by MEXT KAKENHI Grants: a Grant-in-Aid for Scientific Research on the Innovative Area of Non-coding DNA (nos 24114516 and 26114717).

\section{Author Contributions}

H.N. designed and performed the experiments, and wrote the manuscript. K.Y. generated strains for protein expression, and purified proteins.

\section{Additional Information \\ Supplementary information accompanies this paper at http://www.nature.com/srep}

Competing financial interests: The authors declare no competing financial interests.

How to cite this article: Niki, H. and Yano, K. In vitro topological loading of bacterial condensin MukB on DNA, preferentially single-stranded DNA rather than double-stranded DNA. Sci. Rep. 6, 29469; doi: 10.1038/ srep29469 (2016).

(c) (i) This work is licensed under a Creative Commons Attribution 4.0 International License. The images or other third party material in this article are included in the article's Creative Commons license, unless indicated otherwise in the credit line; if the material is not included under the Creative Commons license, users will need to obtain permission from the license holder to reproduce the material. To view a copy of this license, visit http://creativecommons.org/licenses/by/4.0/ 